\title{
Quantum Chemistry Estimation of Adhesion Strength of Hydroxyapatite with Titanium Substrate
}

Alla V. Balueva ${ }^{1,}{ }^{*}$, Ilia N. Dashevskiy ${ }^{2}$, Patricia Todebush ${ }^{3}$

1. Mathematics Department, University of North Georgia, P.O. Box 1358, Gainesville, Georgia 30503, USA; E-Mail: Alla.Balueva@ung.edu

2. Ishlinsky Institute for Problems in Mechanics RAS, pr. Vernadskogo, 101-1, 119526 Moscow, Russia; E-Mail: dash@ipmnet.ru

3. Chemistry Department, University of North Georgia, P.O. Box 1358, Gainesville, Georgia 30503, USA; E-Mail: Patricia.Todebush@ung.edu

* Correspondence: Alla V. Balueva; E-Mail: Alla.Balueva@ung.edu

Academic Editor: Eugeniusz Sajewicz

Special Issue: Advanced Dental Materials

Recent Progress in Materials

2021, volume 3, issue 4

doi:10.21926/rpm.2104043
Received: May 12, 2021

Accepted: October 14, 2021

Published: October 27, 2021

\begin{abstract}
One of the ways to improve the fusion of an implant with bone tissue is through the use of biocompatible coatings, in particular, hydroxyapatite (HAp). It is important to assess the strength of the HAp adhesion to the implant. The measure of the strength of the bond of the coating with the substrate is the energy of this bond. Using density functional theory and molecular dynamics, the reaction path, reaction products, oscillation frequency, activation energy and bond energy between different combinations of component anions HAp and Ti (II) - the standard material of implants - are calculated. Using the computational chemistry software suite Gaussian 09 (Revision C.01 was used), the stable configurations of the reactants and products are found, and the binding energy of hydroxyapatite and titanium is then calculated based on the difference in ground energy of reactants and ground energy of products. Thus, the method of adhesion strength estimation between Hap coatings and $\mathrm{Ti}$ is
\end{abstract}

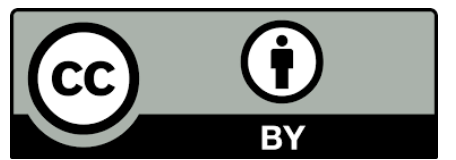

(c) 2021 by the author. This is an open access article distributed under the conditions of the Creative Commons by Attribution License, which permits unrestricted use, distribution, and reproduction in any medium or format, provided the original work is correctly cited. 
proposed based on numerical calculations using MD software, and suggestions are provided on which conditions would be the best for optimal binding strength.

\section{Keywords}

Hydroxyapatite; titanium; surface physics; density functional theory; molecular dynamics; bio nano-coatings; implants

\section{Introduction}

Strong osseointegration between an implant and the host bone tissue is the key to the long-term success of the aforementioned implant (Figure 1) (e.g., [1, 2]). Studies have shown that three key characteristics of an implant surface determine the degree and rate of osseointegration. These characteristics are as follows: mechanical, topological, physiochemical. Titanium (and several of its alloys) has exhibited strong mechanical properties that make it preferable for bone implants [3], but recent work has aimed to improve the topological and biological properties of these implants in the form of bio-nano coatings applied to the surface of a Ti- based implant (e.g., [4-6]). Hydroxyapatite (HAp) with the chemical formula $\mathrm{Ca}_{10}\left(\mathrm{PO}_{4}\right)_{6}(\mathrm{OH})_{2}$ (e.g., [7-9]), a specific chain of the broader Calcium-Phosphate ( $\mathrm{CaP}$ ) family of compounds, has been in wide use as a direct coating to a $\mathrm{Ti}$ implant for over a decade. The coating encourages osseointegration because of its bio-chemical similarity to host bone tissue; however, problems have been identified with its application. Traditionally, the HAp coating has been applied via thermal plasma-spraying (e.g., [10-14]) or via plasma and magnetron sputtering $[15,16]$, which results in suboptimal thickness, inconsistent phases with varying degrees of solubility, and inadequate crystallinity. Also a diffusion method of application of Hap to titanium was used [17], with incorporation of magnesium (Mg) into mineral phase of a scaffold.

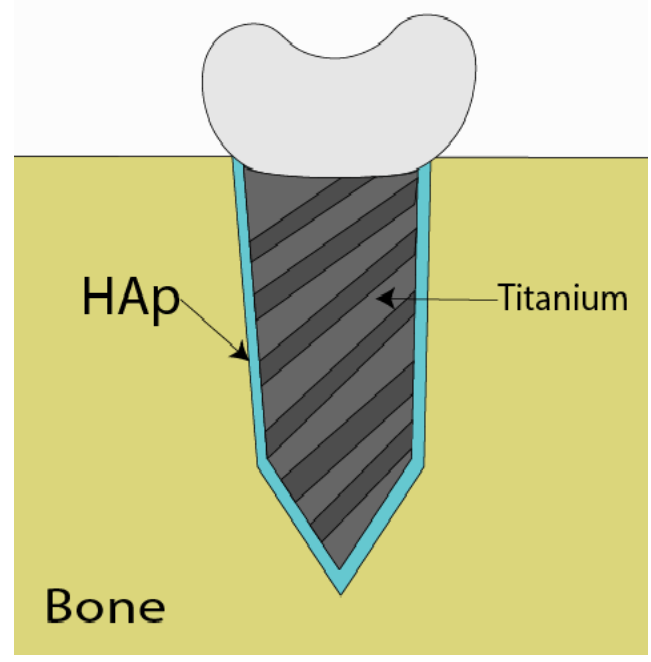

Figure 1 General view of a dental implant screwed into the jaw. 
Since cases of delamination were observed in the clinic at the implant-coating interface [18], the study of the adhesion strength of HAp with titanium is an urgent problem. The characteristic of this strength is the binding energy between titanium and HAp. The purpose of this work was to determine the bond energy between functional groups (anions) of hydroxyapatite and titanium $\mathrm{Ti}$ (II) [19] using computational quantum chemistry (computational chemistry package Gaussian 09, Rev. C.01 [20]).

There are some experimental research on the strength of the nanocoating with the substrate [21-23], although only a few experiments are accounted for because of the complexity of such fine detail at the nanometric level. However, with the advent of DFT and parallel fast computers, Modern Computational Physical Chemistry provides us new possibilities and methods to explore chemical reactions on the boundary of $\mathrm{Ti}$ and $\mathrm{Hap}$, and investigate chemical processes and strength on $a b$ initio, or atomistic level. Titanium is chosen for implants because of its said properties. Hap is chosen for the coating because of its similarity to the structure of bones. Therefore, we cannot change the chemical reactants. Nonetheless, how would they react? Would they adhere well onto each other? This is the main question that we are answering based on computations using methods of Modern Computational Physical Chemistry, which would complement laborious and expensive experiments.

Since the Hap coating is applied to titanium as a nano layer, the methods of DFT can be applied to the physics study of the processes during this reaction. Moreover, this topic attracted attention from the research audience, although only a few articles are available [19, 24-30]. In Jami and Jabbarzadeh [28-30] molecular dynamics (MD) simulations were used to find mechanical deformation, stress and temperature during the fast impact of a $3 \mathrm{~nm}$ HAp particle on a titanium surface. The same authors [31] conducted computation research on deposition of (TiO2) nanoparticles on a titanium (Ti) substrate, using MD simulation with embedded potentials. Depending on velocities of deposition, the temperature and stresses of the final compounds were calculated. In Grubova et. al. [26], the bonding mechanism between HAp coating and a rutile $\left(\mathrm{rTiO}_{2}\right)$ substrate was investigated through Density Functional Theory (DFT), using the PBE (Perdew-BurkeErnzerhof) functional. VASP code was used to simulate the interaction between different phases of titanium dioxide $\left(\mathrm{TiO}_{2}\right)$ and amorphous HAp. Grubova et. al. [26] discovered that of all the different forms of HAp and TiO2, amorphous HAp (aHA) and amorphous titanium dioxide (aTiO2) form on the interface with the strongest adhesion. In Grubova et. al. [27], ab-initio calculations were done to explore the adhesion strength between a-HAp and titanium dioxide with substitution of a P atom by $\mathrm{Si}(\mathrm{a}-\mathrm{Si}-\mathrm{HAP})$, which proved from numerical experiment, creates stronger adhesion between the coating and the substrate due to formation of $\mathrm{OH}$-vacancies [27].

In this research, we use $a b$ initio calculations to investigate the physics aspect of the reactions between hydroxyapatite functional groups (anions) and Ti(II) (titanium cation with plus 2 charge). Specifically, the binding energy of different combinations of the constituents of the hydroxyapatite coating with titanium are calculated and ranked in order of intermolecular interactions and stability of the final products. Using Density Functional Theory, the minimum potential energy was calculated, and the reactants were set up into minimum energy state. Then using the software complex of computational chemistry Gaussian 09, Revision C.01 [20], with the B3LYP functional and basis set of 6-31G, the geometric optimized structures for the reactants were calculated, and the positions of nuclei that deliver the ground state energy were found. The total energy of the system during the reaction decreased since the bonds on the surface between Ti and the coating formed. 
The difference between the ground energy of the reactants and the ground energy of the products gives the binding energy of the coating with the titanium [24].

\section{Computational Method}

We used Density Functional Theory [32, 33] to describe the chemical reaction of different configurations of HAp component ions and Titanium and to determine their geometric and chemical properties. By using the B3LYP hybrid-exchange-correlation functional (Becke three-parameter LeeYang-Parr functional), we determined the ground state energy of polyatomic complexes in the Ti (II) -hydroxyapatite system. The goal is a theoretical calculation of the energy of bonds of the HAp and titanium coatings.

\subsection{Density Functional Theory Basics}

Density functional theory is a theory of approximations on the quantum level that allow a novel method of solving Schrodinger's equation. Instead of relying on solving for the wave function directly, through a series of approximations, we can make the wave function dependent on the electron density functional, a calculable functional [34]. Let us begin with the time-independent Schrodinger equation, which describes the interactions of $N$ particles. It states that when the Hamiltonian, $\hat{H}$, acts on a wave function, $\psi$, it is proportional to the stationary state of the same wave function with a proportionality constant $E$ (which is the total energy for the system):

$$
\widehat{H} \psi\left(x_{1}, x_{2}, \ldots x_{N}\right)=E \psi\left(x_{1}, x_{2}, \ldots x_{N}\right),
$$

The Hamiltonian operator, $\hat{H}$, can be expressed by

$$
\widehat{H}=T^{e l e c}(r)+T^{n u c}(R)+V^{n u c-e l e c}(R, r)+V^{n u c-n u c}(R)+V^{\text {elec-elec }}(r) .
$$

Multiplying Eg. (2.1) by $\overleftarrow{\psi}^{\text {elec }}$

$$
\overleftarrow{\Psi}^{\text {elec }} \widehat{H}^{\text {elec }} \Psi^{\text {elec }}(\vec{r}, \vec{R})=E^{e f f}(\vec{R}) \bar{\Psi}^{\text {elec }} \Psi^{\text {elec }}
$$

and taking into account the normalization of wave functions, this expression can be rewritten as

$$
\overleftarrow{\Psi}^{\text {elec }} \widehat{H}^{\text {elec }} \Psi^{\text {elec }}(\vec{r}, \vec{R})=E^{\text {eff }}
$$

which means the minimization of quadratic functional with respect to $\bar{\psi}^{\text {elec }}$ will give us the true energy minimum, $E^{e f f}$.

In density functional theory, the electron density, $\rho(r)$, is introduced as

$$
\rho(r)=2 \sum_{i}^{N / 2} \psi_{i}(r) \psi_{i}(r)
$$

and the quadratic functional then rewritten in terms of $\rho(r)$ and minimized with respect to the electron density $[35,36]$. 


\subsection{Method of Calculations.}

We found the reaction path, reaction products, oscillation frequency, activation energy, and bond energy between different combinations of component anions of $\mathrm{HAp}$, namely $\mathrm{OH}^{-}, \mathrm{PO}_{4}{ }^{3-}$, and $\mathrm{Ti}^{2+}$. For this, we first set up the reactants $\mathrm{OH}^{-}, \mathrm{PO}_{4}{ }^{3-}$, and $\mathrm{Ti}^{2+}$ into local minima on the potential energy surface (PES) and found their stable configurations and ground state energies. Then, the reaction products of titanium and the constituents of the HAp coating were set up in a stable configuration on the PES (e.g., [34]), and the ground state energies for the products were found. To verify the convergence of the process to the local minimum on the $3 \mathrm{~N}$ surface ( $\mathrm{N}$ is the number of nuclei), we check control of the frequencies of the oscillations at the final stage. If the frequencies are negative, then the results are not a minimum and calculations are repeated from step zero, choosing a different initial configuration of nuclei.

First, for simplicity and optimization of reactants and products and frequency calculations we used a basis set $321 \mathrm{G}$, which gives convergence and positive frequencies. We calculated the final optimized structures (See Figures 2-5), single point energies, bond lengths, bond angles, dihedral angles, point charges and overall electrostatic distributions to understand the interactions of these substances and how they contribute to the process of Osteointegration. Then, we performed the same calculations with a more sophisticated basis set $6-31 G+\left(d^{\prime}\right)$, which requires a longer calculation time but gives more exact binding energies. In Table 1, we introduced the single point energies of different constituents of a hydroxyapatite coating with titanium base of an implant, calculated with basis sets $321 \mathrm{G}$, and $631 \mathrm{G}+\left(\mathrm{d}^{\prime}\right)$ respectively, and their relative errors. We can see that the error of simplification (and taking a simpler basis set $321 \mathrm{G}$ instead of $631 \mathrm{G}+\left(d^{\prime}\right)$ ) increases with complexity of the structure of HAp coating.

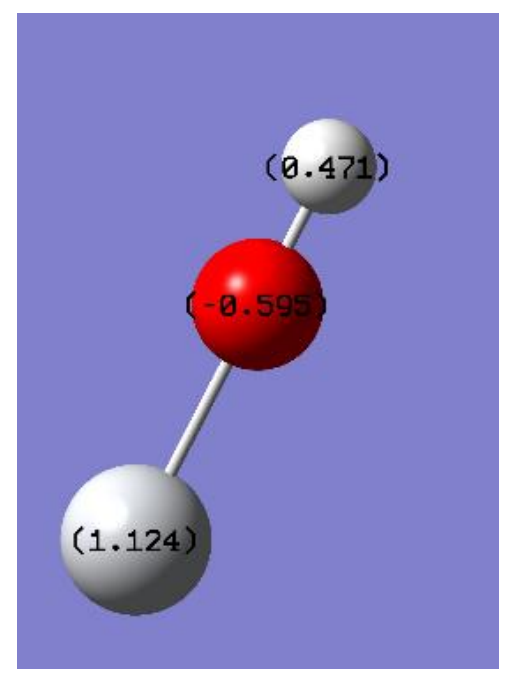

Figure 2 The product of the reaction between $\mathrm{OH}^{-}$and $\mathrm{Ti}^{2+}$. The big grey atom is $\mathrm{Ti}$, the red atom oxygen, and the small grey atom hydrogen. Binding energy is .734 a.u. The charge of the Ti (big grey atom) reduced from $2+$ to +1.124 . 


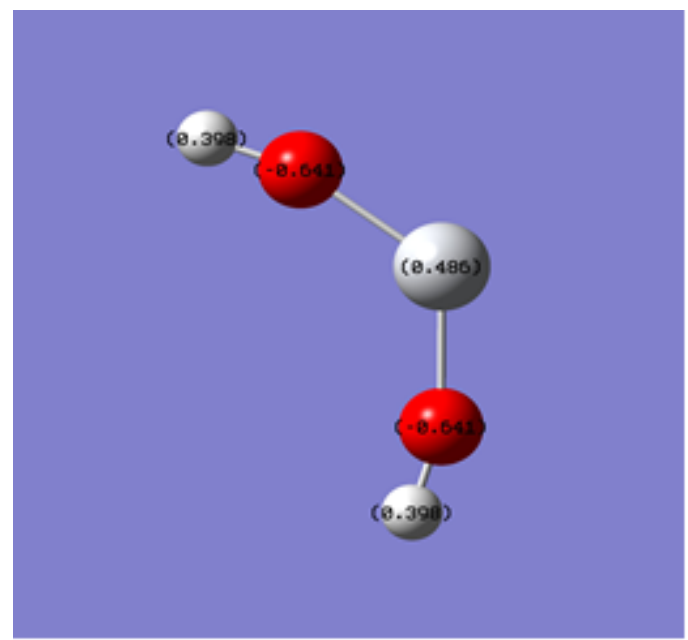

Figure 3 Stable configuration of the product of the reaction between two anions $\mathrm{OH}^{-}$ and $\mathrm{Ti}^{2+}$ in the $\mathrm{Ti}(\mathrm{OH})_{2}$. Binding energy is $1.28 \mathrm{a}$.u. The charge of the Ti (big grey atom) reduced from $2+$ to +0.486 .

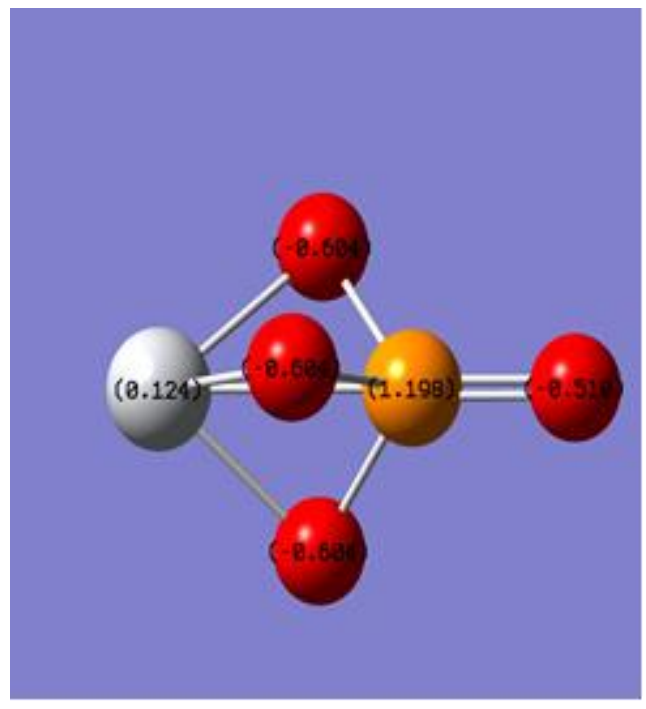

Figure 4 The product of the reaction between $\mathrm{PO}_{4}{ }^{3-}$ and $\mathrm{Ti}^{2+}$. Grey big atom is titanium (with charge +0.124 ), orange atom is Phosphorus (with charge +1.198 ), 4 red atoms are oxygen. Binding energy is 1.88 a.u. The charge of the Ti (big grey atom) reduced from $2+$ to +0.124 . 


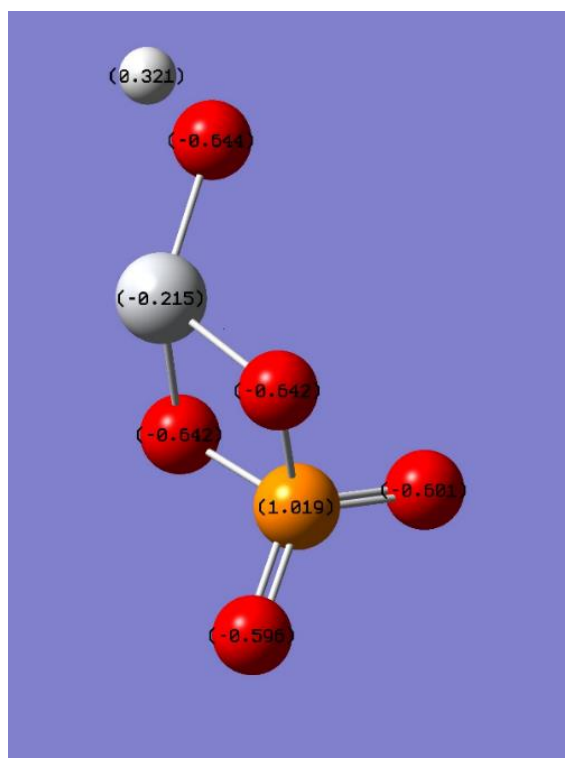

Figure 5 Product of the reaction of $\mathrm{HAp}$ component anions $\mathrm{OH}^{-}, \mathrm{PO}_{4}{ }^{3-}$, and $\mathrm{Ti}^{2+}$. Grey big atom is Ti (with charge -0.215), orange atom is Phosphorus (with charge +1.019), 4 red atoms are oxygen, small grey atoms are hydrogen. The charge of the $\mathrm{Ti}$ (big grey atom) reduced from $2+$ to -0.215 , and so it becomes negative. Whereas the phosphorus charge does not change so dramatically and is still positive, +1.019 . The binding energy for this complex is the greatest, 2.09 a.u.

Table 1 Comparison of single point energy of different polyatomic complexes calculated using different DFT basis sets.

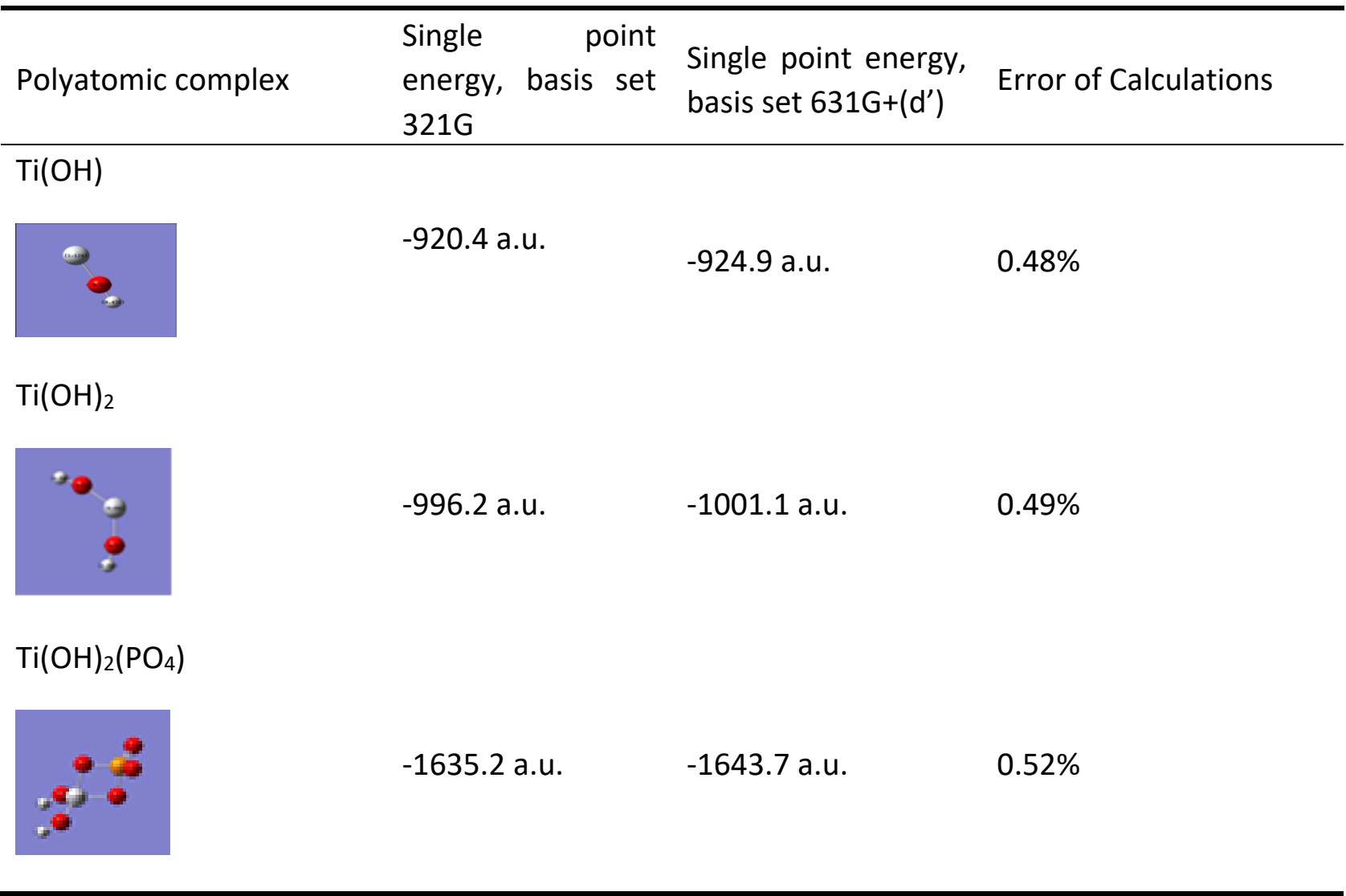




\section{Results of Calculation}

All calculations for the polyatomic complexes in the $\mathrm{Ti}$ (II) -hydroxyapatite system were performed using the DFT functional R3LYP in Gaussian 09 system. These images (Figures 2-5) demonstrate the geometry of the products and therefore give insight into the functionality of the compounds.

The bond energies between individual constituents of HAp and Ti were obtained. For all final geometric structures after the reaction, the equilibrium angles and lengths of the $\mathrm{Ti}-\mathrm{O}$ bonds, electron density distributions, and the ground state energy levels were calculated.

All the structures were ranked in order of binding strength and intermolecular stability. The results are shown in Figures 2-5. Titanium atoms are big grey spheres, hydrogen atoms are small grey spheres, phosphorus atoms are orange spheres, oxygen atoms are red spheres. For all calculations, we need an electrostatic attraction between ions, so we take a cation $\mathrm{Ti}^{2+}$ and our polyatomic constituents participating in the reactions, $\mathrm{OH}^{-}, \mathrm{PO}_{4}{ }^{3-}$, which are all anions.

According to calculations of the complex $\left[\mathrm{Ti}(\mathrm{OH})_{2}\right]$ (Figure 3), the charge of the $\mathrm{Ti}$ (II) ion decreases, from $2+$ to $0.486+$ (a big grey atom in the middle). In terms of the Density Functional Theory (DFT), this means that $\mathrm{Ti}^{2+}$ experiences an increase in the electron density due to its attraction with two negatively charged hydroxide ions, $\mathrm{OH}^{-}$. The Ti-O bond length is about $1.73 \AA$. The Ti-O bond angle is $126^{\circ}$, eigenvalues of Hessian index are positive, which gives real vibrational frequencies of a bond within molecules.

We can see from Figure 4 that the charge of $\mathrm{Ti}$ ion for the complex [ $\left.\mathrm{TiPO}_{4}\right]^{-}$became even less, 0.124 a.u., and for the complex $\left[\mathrm{Ti}(\mathrm{OH}) \mathrm{PO}_{4}\right]^{2-}$ it becomes negative, -0.215 . (Figure 5). The charge of another big ion in the component, phosphate, stays almost the same, without changing. This shows the redistribution of electron density from oxygen atoms in favor of $\mathrm{Ti}$, which makes the configuration more and more stable with its complexity, and also with the number of oxygen atoms in the configuration.

From Figure 2 we can see that $[\mathrm{TiOH}]^{+}$is a linear structure. The repulsion between the positively charged atoms of titanium and hydrogen are likely causing the linearity.

For a complex $\mathrm{Ti}(\mathrm{OH})_{2}$ (Figure 3), higher frequencies are calculated between the titanium and oxygen atoms compared to $[\mathrm{TiOH}]^{+}$. The bond angle is slightly larger due to the oxygen-oxygen repulsion of $\mathrm{Ti}(\mathrm{OH})_{2}$. Additionally, the bond length between the oxygen atoms and the titanium atom has decreased compared to the $[\mathrm{TiOH}]^{+}$structure, which suggests a more favorable higher binding energy for the titanium atom.

In polyatomic complex [ $\left.\mathrm{TiPO}_{4}\right]^{-}$(Figure 4), one of the oxygen atoms is not paired with the titanium atom (big grey atom) and instead develops a stronger and shorter bond to the phosphorus atom (orange) during the interaction. Electron density is shifted towards the $\mathrm{Ti}^{2+}$ atom in this interaction.

In the last complex, $[\mathrm{Ti}(\mathrm{OH}) \mathrm{PO} 4]^{2-}$, when compared to the standard vibrational behavior of phosphate, an increase in frequency between the titanium and oxygen is observed (Figure 5). This interaction suggests a higher energy interaction. Additionally, the bond lengths between titanium and oxygen have shortened, also suggesting a more favorable binding energy.

\section{Discussions}

After it reacts with hydroxide (Figure 2 ), the $\mathrm{Ti}^{2+}$ ion in the $[\mathrm{TiOH}]^{+}$structure experiences a decrease of almost half of its initial charge as observed in its charge distribution. In the complex 
$\left[\mathrm{TiPO}_{4}\right]^{-}$, the $\mathrm{Ti}^{2+}$ experiences an even greater decrease of charge due to the electrons coming from three oxygen atoms (Figure 4). The three oxygen atoms develop a strong bond with the titanium (as observed with a shorter bond length) while the 4th oxygen atom is attracted to the phosphorus atom in $\left[\mathrm{TiPO}_{4}\right]^{-}$.

The shorter bond length between oxygen and titanium in the $\mathrm{Ti}(\mathrm{OH})_{2}$ structure (Figure 3) suggests a stronger bonding interaction between titanium and oxygen compared to the $\left[\mathrm{TiOH}^{\mathrm{t}}\right.$ structure. This suggestion is also supported by the stronger binding energy in $\mathrm{Ti}(\mathrm{OH})_{2}$ compared to the binding energy of $[\mathrm{TiOH}]^{+}$. The increase in binding energy is demonstrated by means of a shorter and stronger bond between the oxygen and titanium due to their electronic exchange.

In the polyatomic complex $\left[\mathrm{Ti}(\mathrm{OH}) \mathrm{PO}_{4}\right]^{2-}$, we observe further lowering of the titanium charge as well as it becoming negative (Figure 5). The titanium atom consistently gains electron density from the oxygen atoms at a dramatic rate, whereas the charge of the phosphorus atom remains almost the same.

Calculations showed that the binding energy increases with each structure due to the increasing electron orbitals around $\mathrm{Ti}$, and, therefore, due to a stronger connection between $\mathrm{Ti}$ and oxygen atoms. Compared to the phosphorus, a titanium atom gains negative electronic charge at much more dramatic rate, whereas the phosphorus charge almost remains without changing. Furthermore, the more complex the structure is, the more negative titanium becomes, which finally creates a centrally negative structure and leads to a lower ground state energy of the products. The lower the ground energy of the products, the higher the binding energy between HAp and titanium is. One can see from Figures 2-5 that, since titanium develops strong electronic connections and forms bonds with surrounding oxygen atoms, there is a limited number of bonds that titanium can form with neighbor phosphate ions. That may give a constraint for the number of the phosphate atoms participating in this reaction, Hydroxyapatite coating - Ti.

\section{Conclusions}

The charge of $\mathrm{Ti}^{2+}$ decreases as the configuration gains more oxygen atoms due to the fact that the electron density around titanium increases at much higher rate than that around the phosphorus. It appears that the most significant factor regarding the strength of the bond of Ti(II) and HAp is the number of oxygen atoms in the structure and their interaction with the titanium atom, which means that the more complex the structure of constituents of HAp, the higher the binding energy between them and titanium. Furthermore, the increase of the electron density of titanium leads to the changing of other properties of structures such as stronger bonds, higher interaction frequencies, and more favorable geometries.

In conclusion, we can say that the calculations demonstrated that the hydroxyapatite and titanium develop "right" binding strength and stable structures, which indicates that hydroxyapatite is not only a favorable material from the point of view of accelerating osteointegration on the boundary bone - HAp, but also a compatible material to have a good adhesion strength on the boundary hydroxyapatite coating - titanium implant.

In dental practices, it is important to take into account that the chemicals that are attached to the implants should not interfere with the healing process. Because of this, it is important to maximize the adhesive strength of nano-coatings to not have unpleasant peeling processes of the coating in the future. This research shows ways of optimizing the structure of the calcium phosphate 
coatings to have their highest adhesive characteristics through means of computer MD calculations, which will significantly reduce the volume, cost and efforts for expensive, scrupulous and, therefore, difficult experiments.

\section{Acknowledgements}

The research was done with partial support of RFBR grants No. 17-08-01579 and No. 17-08-01312 and of contract \#AAAA-A17-117021310386-3. This work used the Extreme Science and Engineering Discovery Environment (XSEDE), which is supported by National Science Foundation grant number $\mathrm{ACl}-1548562$ [37]. We thank consultants for their assistance with porting, optimization and visualization, which was made possible through the XSEDE Extended Collaborative Support Service (ECSS) program [38]. Authors also thank UNG students Chasen Campbell, Eduardo Valdez and Wynn Kwiatkowskiy for help with calculations.

\section{Author Contributions}

Alla Balueva: Methodology, Validation, Writing-Original draft preparation, Reviewing and Editing Ilia Dashevskiy: Conceptualization, Methodology, Supervision Patricia Todebush: Data curation, Recourses

\section{Competing Interests}

The authors have declared that no competing interests exist.

\section{References}

1. Albrektsson $\mathrm{T}$, Johansson C. Osteoinduction, osteoconduction and osseointegration. Eur Spine J. 2001; 10: S96-S101.

2. Swetha M, Sahithi K, Moorthi A, Srinivasan N, Ramasamy K, Selvamurugan N. Biocomposites containing natural polymers and hydroxyapatite for bone tissue engineering. Int $\mathrm{J}$ Biol Macromol. 2010; 47: 1-4.

3. Huang CF, Chiang HJ, Lin HJ, Hosseinkhani H, Ou KL, Peng PW. Comparison of cell response and surface characteristics on titanium implant with SLA and SLAffinity functionalization. J Electrochem Soc. 2014; 161: G15.

4. Xuereb M, Camilleri J, Attard NJ. Systematic review of current dental implant coating materials and novel coating techniques. Int J Prosthodont. 2015; 28: 51-59.

5. Menicucci G, Mussano F, Schierano G, Rizzati A, Aimetti M, Gassino G, et al. Healing properties of implants inserted concomitantly with anorganic bovine bone. A histomorphometric human study. Aust Dent J. 2013; 58: 57-66.

6. Vladescu A, Surmeneva MA, Cotrut CM, Surmenev RA, Antoniac IV. Bioceramic coatings for metallic implants. In: Handbook of bioceramics and biocomposites. Switzerland: Springer; 2016.

7. Eliaz N, Metoki N. Calcium phosphate bioceramics: A review of their history, structure, properties, coating technologies and biomedical applications. Materials. 2017; 10: 334.

8. Dorozhkin SV. Nanodimensional and nanocrystalline hydroxyapatite and other calcium orthophosphates. In: Hydroxyapatite: Synthesis, properties and applications. Hauppauge, New York: Nova Science Publishers; 2012. 
9. Surmenev RA, Surmeneva MA, Ivanova AA. Significance of calcium phosphate coatings for the enhancement of new bone osteogenesis-a review. Acta Biomater. 2014; 10: 557-579.

10. Surmenev RA. A review of plasma-assisted methods for calcium phosphate-based coatings fabrication. Surf Coat Technol. 2012; 206: 2035-2056.

11. Jansen JA, Leon B. Thin calcium phosphate coatings for medical implants. New York: Springer; 2009.

12. Bigi A, Bracci B, Cuisinier F, Elkaim R, Fini M, Mayer I, et al. Human osteoblast response to pulsed laser deposited calcium phosphate coatings. Biomaterials. 2005; 26: 2381-2389.

13. Roy $M$, Bandyopadhyay A, Bose $S$. Induction plasma sprayed nano hydroxyapatite coatings on titanium for orthopaedic and dental implants. Surf Coat Technol. 2011; 205: 2785-2792.

14. Ou SF, Chen CS, Hosseinkhani H, Yu CH, Shen YD, Ou KL. Surface properties of nano-structural silicon-doped carbon films for biomedical applications. Int J Nanotechnol. 2013; 10: 945-958.

15. Ou KL, Chu JS, Hosseinkhani H, Chiou JF, Yu CH. Biomedical nanostructured coating for minimally invasive surgery devices applications: Characterization, cell cytotoxicity evaluation and an animal study in rat. Surg Endosc. 2014; 28: 2174-2188.

16. Ou KL, Hosseinkhani H. Development of 3D in vitro technology for medical applications. Int J Mol Sci. 2014; 15: 17938-17962.

17. Baheiraei N, Azami M, Hosseinkhani H. Investigation of magnesium incorporation within gelatin/calcium phosphate nanocomposite scaffold for bone tissue engineering. Int J Appl Ceram Technol. 2015; 12: 245-253.

18. Subramani K, Mathew R, Hosseinkhani $H$, Hosseinkhani $M$. Bone regeneration around dental implants as a treatment for peri-implantitis: A review of the literature. J Biomim Biomater Tissue Eng. 2011; 11: 21-33.

19. Balueva AV, Dashevskiy IN, Todebush P, Campbell C, Valdez E. First-principle calculations of the binding energy of the coating components of new generation dental implants. Proceedings of the ASME 2019 International Mechanical Engineering Congress and Exposition; 2019 November 11-14; Salt Lake City, Utah, USA.

20. Ruys A, Wei M, Sorrell C, Dickson M, Brandwood A, Milthorpe B. Sintering effects on the strength of hydroxyapatite. Biomaterials. 1995; 16: 409-415.

21. Lindström S, lles A, Persson J, Hosseinkhani H, Hosseinkhani M, Khademhosseini $A$, et al. Nanoporous titania coating of microwell chips for stem cell culture and analysis. J Biomech Sci Eng. 2010; 5: 272-279.

22. Shahrezaei M, Habibzadeh S, Babaluo AA, Hosseinkhani H, Haghighi M, Hasanzadeh A, et al. Study of synthesis parameters and photocatalytic activity of $\mathrm{TiO}_{2}$ nanostructures. J Exp Nanosci. 2016; 12: 45-61.

23. Balueva AV, Dashevskiy IN. Atomistic modeling and strength calculations of Ni-Al submicron and nanosized composites. Proceedings of the 7th International Conference on Mechanics and Materials in Design; 2017 June 11-15; Albufeira, Portugal.

24. Balueva AV, Dashevskiy IN, Todebush P, Campbell C. Modeling bonding energy in novel bioactive nano coatings on dental implants. Procedia Struct Integr. 2019; 23: 173-178.

25. Grubova IY, Surmeneva MA, Huygh S, Surmenev RA, Neyts EC. Density functional theory study of interface interactions in hydroxyapatite/rutile composites for biomedical applications. J Phys Chem C. 2017; 121: 15687-15695. 
26. Grubova IY, Surmeneva MA, Huygh S, Surmenev RA, Neyts EC. Effects of silicon doping on strengthening adhesion at the interface of the hydroxyapatite-titanium biocomposite: $A$ firstprinciples study. Comput Mater Sci. 2019; 159: 228-234.

27. Jami $H$, Jabbarzadeh $A$. Ultrafast thermomechanical effects in aerosol deposition of hydroxyapatite nanoparticles on a titanium substrate. Surf Coat Technol. 2020; 382: 125173.

28. Jami $\mathrm{H}$, Jabbarzadeh $\mathrm{A}$. Unravelling ultrafast deformation mechanisms in surface deposition of titanium nanoparticles. Appl Surf Sci. 2019; 489: 446-461.

29. Jami $\mathrm{H}$, Jabbarzadeh $\mathrm{A}$. Effect of particle shape on mechanics of impact in the deposition of titanium nanoparticles on a titanium substrate. Surf Coat Technol. 2020; 394: 125880.

30. Jami $\mathrm{H}$, Jabbarzadeh $\mathrm{A}$. Molecular simulation of high-velocity deposition of titanium dioxide nanoparticles on titanium. Appl Surf Sci. 2021; 542: 148567.

31. Frisch MJ, Trucks GW, Schlegel HB, Scuseria GE, Robb MA, Cheeseman JR, et al. Gaussian 09, Revision A.02. Wallingford CT: Gaussian, Inc.; 2016. Available from: http://www.gaussian.com/.

32. Laird BB, Ross RB, Ziegler T. Chemical applications of density-functional theory. Washington, DC: ACS Publications; 1996.

33. Koch W, Holthausen MC. A chemist's guide to density functional theory. Weinheim: Wiley VCH; 2001.

34. Carr JM, Trygubenko SA, Wales DJ. Finding pathways between distant local minima. J Chem Phys. 2005; 122: 234903.

35. Kohn W, Holthausen M. A chemist's guide to density functional theory. Weinheim: Wiley-VCH Verlag $\mathrm{GmbH} ; 2001$. pp.295.

36. Laird BB, Ross RB, Ziegler T. Chemical applications of density functional theory. Washington, DC: American Chemical Society; 1996.

37. Towns J, Cockerill T, Dahan M, Foster I, Gaither K, Grimshaw A, et al. XSEDE: Accelerating scientific discovery. Comput Sci Eng. 2014; 16: 62-74.

38. Wilkins-Diehr N, Sanielevici S, Alameda J, Cazes J, Crosby L, Pierce M, et al. High performance computer applications. Proceedings of the 6th International Conference, ISUM; 2015 March 913; Mexico City, Mexico.

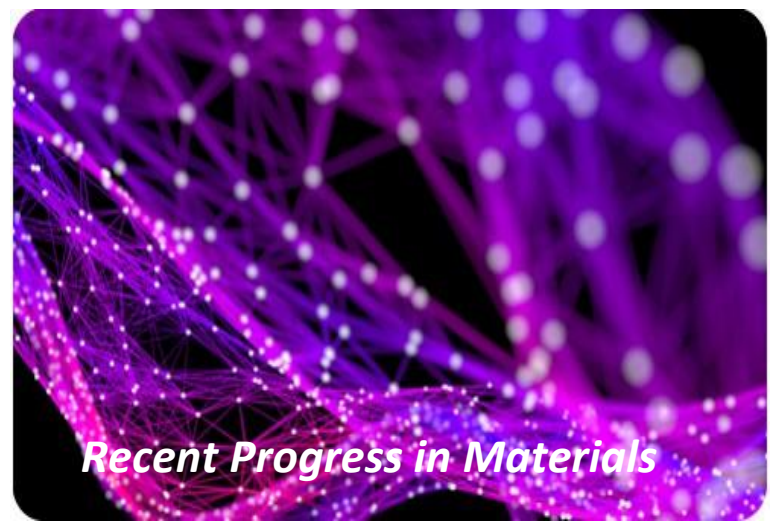

Enjoy Recent Progress in Materials by:

1. Submitting a manuscript

2. Joining in volunteer reviewer bank

3. Joining Editorial Board

4. Guest editing a special issue

For more details, please visit: http://www.lidsen.com/journals/rpm 